\title{
Fractal scales in a Schwarzschild atmosphere
}

\author{
E N Glass $†$ and J P Krisch \\ Department of Physics, University of Michigan, Ann Arbor, MI 48109, USA \\ Received 17 November 1999, in final form 22 May 2000
}

\begin{abstract}
Recently, Glass and Krisch have extended the Vaidya radiating metric to include both a radiation fluid and a string fluid (1999 Class. Quantum Grav. 16 1175). Mass diffusion in the extended Schwarzschild atmosphere was studied. The continuous solutions of classical diffusive transport are believed to describe the envelope of the underlying fractal behaviour. In this work we examine the classical picture at scales on which fractal behaviour might be evident.
\end{abstract}

PACS numbers: 0420J, 545D, 646

\section{Introduction}

Since its discovery in 1916, the Schwarzschild vacuum solution has been a source of many insights into relativistic physics.

$$
g_{a b}^{S c h} \mathrm{~d} x^{a} \mathrm{~d} x^{b}=\left(1-2 m_{0} / r\right) \mathrm{d} u^{2}+2 \mathrm{~d} u \mathrm{~d} r-r^{2} \mathrm{~d} \Omega^{2} .
$$

This solution and its extensions contain new views of relativistic physics on a variety of scales, from the size of the Universe down to the size of a stellar black hole. When contemplating quantum effects at classical boundaries such as the Schwarzschild horizon, the scales become much smaller down to the Planck length $L_{p}=\sqrt{\hbar G / c^{3}}=1.6 \times 10^{-33} \mathrm{~cm}$. The classical point particle electron may perhaps be resolved into Planck scale strings bits with size proportional to the Planck length and with $10^{20}$ strings bits fitting into a classical electron radius.

Vaidya [1] extended the solution by allowing the Schwarzschild mass parameter to be a function of retarded time, $m(u)$, and discovered a spherically symmetric null fluid atmosphere beyond the horizon at $2 m_{0}$. Glass and Krisch (GK) [2] pointed out that allowing the Schwarzschild mass parameter to be a function of radial position and retarded time, $m(u, r)$, creates an atmosphere with both a null fluid and string fluid stress-energy content described by a metric $g_{a b}^{G K}$. The density of the string fluid, $\rho$, obeys a classical diffusion equation in the Schwarzschild spacetime:

$$
\partial_{u} \rho=r^{-2} \partial_{r}\left(D r^{2} \partial_{r} \rho\right)
$$

where $D(r)$ is the variable diffusivity [3] found in anomalous diffusion. One may show [4], if the mass is diffusing in a flowspace with $\sqrt{-g}=r^{-\beta} f(\vartheta)$ (in [4] $\beta=1-\delta$ ), that the functional dependence of the diffusivity is determined,

$$
\begin{aligned}
& \partial_{u} m=r^{\beta} \partial_{r}\left(D r^{-\beta} \partial_{r} m\right) \\
& D(r)=D_{0} r^{\beta-2} .
\end{aligned}
$$

$\dagger$ Permanent address: Physics Department, University of Windsor, Ontario, Canada N9B 3P4. 
The continuous solutions of classical diffusive transport are believed to describe the envelope of the underlying fractal behaviour [5]. Fractal behaviour is usually associated with mass scaling but the radial dependence of the diffusivity [6] carries additional information about the connectivity of the underlying fractal substrate, and so we wish to examine the classical picture at scales on which fractal behaviour might be evident. These smaller scales are still classical (i.e. larger than quantum scales). We consider a two-parameter family of spacetimes with the metric function $A=1-2 m(u, r) / r$

$$
g_{a b}^{\text {scale }} \mathrm{d} x^{a} \mathrm{~d} x^{b}=A \mathrm{~d} u^{2}+2 \mathrm{~d} u \mathrm{~d} r-\lambda^{2-\alpha} r^{\alpha} \mathrm{d} \Omega^{2},
$$

where $\lambda$ has units of length, and is required for spheres to have an area of (length) ${ }^{2}$. This metric is capable of describing different scales for a particular central source. For example, a particular mass function $m(u, r)$ with $\alpha=2$, could describe the Schwarzschild vacuum outside the source. For $\alpha \neq 2$ it would describe the source at different scales where we expect evidence of fractal behaviour to appear. Fractal dimensions are generally less than the Euclidean dimension, and we will describe an averaging process on 2-spheres for $\alpha \leqslant 2$. The scale parameter $\alpha$ cannot be removed by a coordinate transformation. If one attempts the appearance of the usual area coordinate by $y^{2}=\lambda^{2-\alpha} r^{\alpha}$, then $\alpha$ will emerge in the $(u, r)$ part of $g_{a b}^{\text {scale }}$. Moreover, the manifestly coordinate-independent sectional curvature of the $(\vartheta, \varphi)$ 2 -surfaces, given in equation (15) below, provides a physically meaningful mass expression in terms of $\alpha$.

The interpretation of $\alpha$ as a fractal scale follows from considering diffusive processes in the atmosphere. If $\mathcal{P}(u, r)$ is a quantity that is diffusing, the diffusion equation is

$$
\partial_{u} \mathcal{P}=r^{-\alpha} \partial_{r}\left(D r^{\alpha} \partial_{r} \mathcal{P}\right)
$$

and $\alpha+1$ is identified as the dimension of the fractal substrate on which the diffusion is occurring [5,7]. At smaller quantum levels, $\mathcal{P}$ can be a probability measure on the space of radial paths [8].

The fluid atmosphere described by this metric is discussed in the next section. In section 3 diffusion and scale sizes are discussed. Definitions of topological and Hausdorff dimensions are given. Applications of the fractal view are presented in section 4, along with specific mass solutions. The paper is summarized in the final section.

In this work Latin indices range over $(0,1,2,3)=(u, r, \vartheta, \varphi)$. Our sign conventions are $2 A_{c ;[a b]}=A_{e} R_{c a b}^{e}$ and $R_{a b}=R_{a b c}^{c}$. Overdots denote $\partial / \partial u$ and primes denote $\partial / \partial r$. Overhead carets denote unit vectors. We use units where $G=c=1$. Einstein's field equations are $G_{a b}=-8 \pi T_{a b}$, and the metric signature is $(+,-,-,-)$.

\section{The atmosphere}

The metric $g_{a b}^{\text {scale }}$ describes an atmosphere that admits a two-fluid description of matter with outward flowing short-wavelength photons (sometimes called a 'null fluid').

With the use of a Newman-Penrose null tetrad the Einstein tensor for the atmosphere is computed from (4) and given by

$$
G_{a b}=-2 \Phi_{11}\left(l_{a} n_{b}+n_{a} l_{b}+m_{a} \bar{m}_{b}+\bar{m}_{a} m_{b}\right)-2 \Phi_{00} n_{a} n_{b}-2 \Phi_{22} l_{a} l_{b}-6 \Lambda g_{a b} .
$$

Here the null tetrad components of the Ricci tensor are

$$
\begin{aligned}
\Phi_{00} & =\frac{\alpha(2-\alpha)}{4 r^{2}}, \\
\Phi_{11} & =\frac{2 m^{\prime}-r m^{\prime \prime}}{4 r^{2}}-\frac{\alpha^{2}}{16 r^{2}}+\frac{\lambda^{\alpha-2}}{4 r^{\alpha}}+\frac{(\alpha-2)(\alpha+2) m}{8 r^{3}},
\end{aligned}
$$


$\Phi_{22}=-\frac{\alpha \dot{m}}{2 r^{2}}+\frac{\alpha(2-\alpha)}{16 r^{2}}(1-2 m / r)^{2}$

$\Lambda=R / 24=\frac{r m^{\prime \prime}+2(\alpha-1) m^{\prime}}{12 r^{2}}+\frac{(\alpha-2)(3 \alpha-2) m}{24 r^{3}}-\frac{\alpha(3 \alpha-4)}{48 r^{2}}+\frac{\lambda^{\alpha-2}}{12 r^{\alpha}}$.

The only non-zero component of the Weyl tensor is

$$
\Psi_{2}=-\frac{(\alpha+1) m}{3 r^{3}}+\frac{(2+\alpha) m^{\prime}-r m^{\prime \prime}}{6 r^{2}}+\frac{\alpha}{12 r^{2}}-\frac{\lambda^{\alpha-2}}{6 r^{\alpha}} .
$$

The metric is Petrov type $\boldsymbol{D}$ with $l^{a}$ and $n^{a}$ principal null geodesic vectors

$$
\begin{aligned}
& l_{a} \mathrm{~d} x^{a}=\mathrm{d} u, \quad l^{a} \partial_{a}=\partial_{r} \\
& n_{a} \mathrm{~d} x^{a}=\frac{1}{2} A \mathrm{~d} u+\mathrm{d} r, \quad n^{a} \partial_{a}=\partial_{u}-\frac{1}{2} A \partial_{r} \\
& m_{a} \mathrm{~d} x^{a}=-(1 / \sqrt{ } 2) \lambda^{1-\alpha / 2} r^{\alpha / 2}(\mathrm{~d} \vartheta+\mathrm{i} \sin \vartheta \mathrm{d} \varphi), \\
& m^{a} \partial_{a}=(1 / \sqrt{2}) \lambda^{\alpha / 2-1} r^{-\alpha / 2}\left(\partial_{\vartheta}+\frac{\mathrm{i}}{\sin \vartheta} \partial_{\varphi}\right) .
\end{aligned}
$$

When $\alpha=2$ all quantities have the spacetime values for the metric $g_{a b}^{G K}$ discussed in [2].

In order to clearly see the matter content we introduce a timelike unit velocity vector $\hat{v}^{a}$ and three unit spacelike vectors $\hat{r}^{a}, \hat{\vartheta}^{a}, \hat{\varphi}^{a}$ such that

$$
g_{a b}^{\text {scale }}=\hat{v}_{a} \hat{v}_{b}-\hat{r}_{a} \hat{r}_{b}-\hat{\vartheta}_{a} \hat{\vartheta}_{b}-\hat{\varphi}_{a} \hat{\varphi}_{b}
$$

The unit vectors are defined by

$$
\begin{array}{llrl}
\hat{v}_{a} \mathrm{~d} x^{a}=A^{1 / 2} \mathrm{~d} u+A^{-1 / 2} \mathrm{~d} r, & & \hat{v}^{a} \partial_{a}=A^{-1 / 2} \partial_{u}, \\
\hat{r}_{a} \mathrm{~d} x^{a}=A^{-1 / 2} \mathrm{~d} r, & \hat{r}^{a} \partial_{a}=A^{-1 / 2} \partial_{u}-A^{1 / 2} \partial_{r}, \\
\hat{\vartheta}_{a} \mathrm{~d} x^{a}=\lambda^{1-\alpha / 2} r^{\alpha / 2} \mathrm{~d} \vartheta, & \hat{\vartheta}^{a} \partial_{a}=-\lambda^{\alpha / 2-1} r^{-\alpha / 2} \partial_{\vartheta}, \\
\hat{\varphi}_{a} \mathrm{~d} x^{a}=\lambda^{1-\alpha / 2} r^{\alpha / 2} \sin \vartheta \mathrm{d} \varphi, & \hat{\varphi}^{a} \partial_{a}=-\lambda^{\alpha / 2-1}\left(r^{\alpha / 2} \sin \vartheta\right)^{-1} \partial_{\varphi} .
\end{array}
$$

The Einstein tensor (6) yields the energy-momentum tensor

$$
T_{a b}=\psi l_{a} l_{b}+\rho \hat{v}_{a} \hat{v}_{b}+p_{r} \hat{r}_{a} \hat{r}_{b}+p_{\perp}\left(\hat{\vartheta}_{a} \hat{\vartheta}_{b}+\hat{\varphi}_{a} \hat{\varphi}_{b}\right)+q_{a} \hat{v}_{b}+\hat{v}_{a} q_{b}
$$

where

$$
\begin{aligned}
& 8 \pi \psi=-\frac{\alpha \dot{m}}{r^{2}}+\frac{\alpha(2-\alpha) A^{2}}{8 r^{2}}, \\
& 8 \pi \rho=\frac{\alpha m^{\prime}}{r^{2}}+\frac{\lambda^{\alpha-2}}{r^{\alpha}}-\frac{\alpha(\alpha-1)}{2 r^{2}}+\frac{(\alpha-2)}{8 r^{3}}[8 \alpha m-\alpha(r-2 m)], \\
& 8 \pi p_{r}=-\frac{\alpha m^{\prime}}{r^{2}}-\frac{\lambda^{\alpha-2}}{r^{\alpha}}+\frac{\alpha(\alpha-1)}{2 r^{2}}-\frac{(\alpha-2)}{8 r^{3}}[8 \alpha m+\alpha(r-2 m)], \\
& 8 \pi p_{\perp}=-\frac{m^{\prime \prime}}{r}-\frac{(\alpha-2) m^{\prime}}{r^{2}}-\frac{(\alpha-2)^{2} m}{2 r^{3}}+\frac{\alpha(\alpha-2)}{4 r^{2}}, \\
& 8 \pi q_{a}=\left[A \frac{\alpha(2-\alpha)}{8 r^{2}}\right] \hat{r}_{a} .
\end{aligned}
$$

The string equation of state $\rho+p_{r}=0$ holds for the scale with $\alpha=2$. The decomposition of the energy-momentum tensor given in equation (11) is less compelling on $\alpha \neq 2$ scales since there is heat flow in addition to the null fluid. The heat flow arises from the $\Phi_{00} n_{a} n_{b}$ term in 
$G_{a b}$. This is seen only on $\alpha \neq 2$ scales and is the intermediate electromagnetic field part of the null fluid, which only has a far-field part in the usual Vaidya large-scale view. A different covariant decomposition using $l_{a} \mathrm{~d} x^{a}=A^{-1}\left(\hat{v}_{a}-\hat{r}_{a}\right) \mathrm{d} x^{a}$ provides a single fluid with radial heat flow. The energy-momentum tensor decomposes as

$$
T_{a b}=\tilde{\rho} \hat{v}_{a} \hat{v}_{b}+\tilde{p}_{r} \hat{r}_{a} \hat{r}_{b}+p_{\perp}\left(\hat{\vartheta}_{a} \hat{\vartheta}_{b}+\hat{\varphi}_{a} \hat{\varphi}_{b}\right)+\tilde{q}_{a} \hat{v}_{b}+\hat{v}_{a} \tilde{q}_{b}
$$

where

$$
\begin{aligned}
& 8 \pi \tilde{\rho}=-A^{-1} \frac{\alpha \dot{m}}{r^{2}}+\frac{\alpha m^{\prime}}{r^{2}}+\frac{\lambda^{\alpha-2}}{r^{\alpha}}-\frac{\alpha(\alpha-1)}{2 r^{2}}+\frac{(\alpha-2)}{4 r^{3}}[4 \alpha m-\alpha(r-2 m)], \\
& 8 \pi \tilde{p}_{r}=-A^{-1} \frac{\alpha \dot{m}}{r^{2}}-\frac{\alpha m^{\prime}}{r^{2}}-\frac{\lambda^{\alpha-2}}{r^{\alpha}}+\frac{\alpha(\alpha-1)}{2 r^{2}}-\frac{(\alpha-2)}{4 r^{3}}[4 \alpha m+\alpha(r-2 m)], \\
& 8 \pi \tilde{q}_{a}=\left(A^{-1} \frac{\alpha \dot{m}}{r^{2}}\right) \hat{r}_{a} .
\end{aligned}
$$

We note that

$$
8 \pi\left(\tilde{\rho}+\tilde{p}_{r}\right)=-A^{-1} \frac{2 \alpha \dot{m}}{r^{2}}-\frac{\alpha(\alpha-2)}{2 r^{3}}(r-2 m) .
$$

In the static case the string equation of state $\tilde{\rho}+\tilde{p}_{r}=0$ holds on the horizon for all $\alpha$. For mass $m(r)$ which falls off at least as fast as $\mathrm{O}\left(1 / r^{2}\right)$, the string equation of state also holds at future null infinity.

The metric function $m(u, r)$ measures the system mass only for $\alpha=2$, the GK metric. In general, spherical symmetry allows the mass within 2-surfaces of constant $u$ and $r$ to be invariantly defined by the sectional curvature [9] of those surfaces:

$$
\begin{aligned}
-2 M / r^{3} & :=R_{a b c d} \hat{\vartheta}^{a} \hat{\varphi}^{b} \hat{\vartheta}^{c} \hat{\varphi}^{d} \\
& =-\frac{\alpha^{2} m}{2 r^{3}}+\frac{\alpha^{2}}{4 r^{2}}-\frac{\lambda^{\alpha-2}}{r^{\alpha}} .
\end{aligned}
$$

When $\alpha=2$ then $M=m$.

\section{Diffusion and scale}

\subsection{Diffusion}

Continuous diffusive processes can envelope underlying fractal behaviour. In the $\alpha=2$ Schwarzschild atmosphere, the density $\rho$ and the mass function $m$ diffuse according to equations (2) and (3). By analogy with the classical diffusion of density in the Schwarzschild spacetime, we assume that the density enveloping fractal behaviour obeys

$$
\partial_{u} \Gamma=r^{-\alpha} \partial_{r}\left(D r^{\alpha} \partial_{r} \Gamma\right)
$$

where the mass density $\Gamma$ is defined on $u=$ constant surfaces, using the sectional curvature mass, by the relation $M=\int \Gamma \sqrt{-g} \mathrm{~d}^{3} x$ so that

$$
4 \pi\left(\Gamma-\Gamma_{0}\right):=\lambda^{\alpha-2} r^{-\alpha} \partial_{r} M .
$$

The relationship between the energy density $\rho$, seen by observer $\hat{v}^{a}$, in (12b) and the mass density is then given by

$$
\begin{aligned}
4 \pi \Gamma=4 \pi \rho+ & \left(\frac{1}{8 r^{2}}\right)\left[(\lambda / r)^{\alpha-2} \alpha^{2}\left(2 m^{\prime}-1\right)-2 \alpha\left(2 m^{\prime}-\alpha+1\right)\right] \\
& +\left(\frac{1}{2 r^{2}}\right)(\lambda / r)^{\alpha-2}\left[(3-\alpha)(\lambda / r)^{\alpha-2}-1\right]-\frac{(\alpha-2)}{16 r^{3}}[8 \alpha m-\alpha(r-2 m)] .
\end{aligned}
$$


In the region where $\alpha=2$, the two densities $\Gamma$ and $\rho$ are equal.

Assumption (16) is equivalent to assuming a Fickian mass current of the form

$$
\partial_{u} M=4 \pi D(r) r^{\alpha} \partial_{r} \Gamma,
$$

and then $\partial_{r} M=4 \pi \lambda^{2-\alpha} r^{\alpha}\left(\Gamma-\Gamma_{0}\right)$ yields (16). Furthermore, if the sectional curvature mass $M$ diffuses as

$$
\partial_{u} M=r^{\beta} \partial_{r}\left(D r^{-\beta} \partial_{r} M\right)
$$

then $D(r)$ is determined by $\partial_{u} M$ in (19) and $\partial_{r} M$ :

$$
D(r)=D_{0} r^{\beta-\alpha} .
$$

Note that if $\alpha=2$ the $M$ diffusion equation is just equation (3) with $\beta=1-\delta$. For any $\alpha$, when $D=D_{0}$, it follows that $\beta=\alpha$ and that $M$ and $\Gamma$ diffuse reciprocally.

Equation (20) admits the homogeneous solution

$$
M_{\text {hom }}(r)=M_{0}+M_{1}(r / \lambda)^{1+\alpha}
$$

which can be added to each time-dependent solution. There are two important observations that follow from these results. First, the metric of $M_{\text {hom }}$ is not asymptotically flat and the diffusive solution would need to be matched to a solution with appropriate asymptotic behaviour. Second, the diffusivity depends on a coordinate and not on the size of any correlated mass grouping. This implies that there may be multiple distance scales in the atmosphere.

\subsection{Dimensions}

To better understand fractal scales we briefly discuss fractal dimensions [10]. We cannot use the intuitive notion of the number of coordinates needed to locate a point in some region as the dimension of the region, since Peano constructed a curve (one coordinate) which fills a square (two coordinates). Consider a point in set $\Omega$, with $\Omega$ a subset of a Euclidean space of integer dimension $d_{E}$. The notion of topological dimension involves the idea that neighbourhoods around a point have boundaries of smaller dimension. Precisely, the topological dimension $d_{T}(\Omega)$ of set $\Omega$ is the smallest integer such that each point has small neighbourhoods with boundaries of lesser dimension. Since $d_{T}(\emptyset)=-1$ it is clear that $d_{T}(\Omega) \leqslant d_{E}$.

The Hausdorff dimension [11] of self-similar sets (fractals) is given in terms of coverings by open sets and is defined as

$$
d_{H}(\Omega):=\lim _{r \rightarrow 0} \frac{-\ln N(r)}{\ln r}
$$

where $N(r)$ is the smallest number of open balls needed to cover $\Omega$. The definition of $d_{H}$ is equivalent to the power law $N(r) \approx$ constant $\times r^{-d_{H}}$. When $d_{H}=3, N(r)$ corresponds to a three-dimensional Newtonian specific density.

The Hausdorff dimension of a fractal set is not always an integer. The scaling dimension $d_{S}$ of fractal sets is more intuitive and, since geometric fractals display regular self-similarity, is equal to the Hausdorff dimension:

$$
d_{S}(\Omega)=\frac{\log (\text { number of replicas })}{\log (\text { magnification factor })} .
$$

Two famous examples are $d_{S}$ (Cantor set) $=\frac{\log (2)}{\log (3)}$ in $d_{E}=1$, and $d_{S}($ Sierpinski gasket $)=\frac{\log (3)}{\log (2)}$ in $d_{E}=2$. An integer-valued example is $d_{S}$ (Sierpinski gasket) $=\frac{\log (4)}{\log (2)}$ in $d_{E}=3$, since all Sierpinski gaskets obey $d_{S}(\Omega)=\frac{\log \left(d_{E}+1\right)}{\log (2)}$. In general, $d_{T}(\Omega) \leqslant d_{H}(\Omega) \leqslant d_{E}$. 


\subsection{Scales}

Maximum scale size is independent of any particular application. Since $\alpha+1$ is the dimension of the fractal substrate, bounded by $d_{E}, \alpha_{\max }=2$.

Consider a vacuum solution at $\alpha=2$ and ask how it is produced. Beginning at a small scale, one considers the atmosphere to be made of many regions. Within each region an average energy density is found; in some of the regions it will be negative, in some positive, and in some zero. Then a larger region, containing several small regions is considered and again an average energy density is produced until in the end we have the vacuum atmosphere with all of the fluctuations averaging to zero. This process is just a renormalization group averaging procedure and, just as in renormalization group calculations on a lattice, it is necessary at each averaging step to rescale. In lattice calculations, one rescales to maintain the lattice spacing. In an atmospheric calculation with a horizon, the rescaling is done to always keep the physical observers outside the horizon. There are several ways in which this could be accomplished.

One method is to have an observer at constant $r$ examine an area of his 2-sphere at different scales while maintaining the same coordinate value of $r$ at all scales. The entire 2 -sphere at $\alpha=2$ can be tiled by elements of area $\delta A_{2}$. The sum of all the tiles is the total area of the sphere $4 \pi r^{2}$. At small scales, the observer chooses a region of the sphere containing many area elements to average over. The result of this averaging must be rescaled at each step, as in lattice renormalization, so that the rescaled area is again $\delta A_{2}$. With this averaging procedure, an observer outside the horizon for $\alpha=2$ will remain outside of the horizon for all $\alpha$. The $\alpha=2$ horizon position will persist at all scales.

To see the effect of the rescaling, consider a typical averaging process. Assume that $\alpha=2$ is the vacuum solution and let the observer look at an area $\delta A_{2}$ and solid angle $\delta \Omega_{2}$ with $\delta A_{2}=r^{2} \delta \Omega_{2}$. This region is the result of averaging over larger regions at the lower scale and then rescaling the area size. The larger region would have an area $\delta A_{\alpha}=\lambda^{2-\alpha} r^{\alpha} \delta \Omega_{\alpha}$. The rescaling would adjust $\delta \Omega_{\alpha}$ to the size of $\delta \Omega_{2}$ and thereby rescale the areas. The rescaling formulae are

$$
\begin{aligned}
& (\lambda / r)^{2-\alpha} \delta \Omega_{\alpha} \longmapsto \delta \Omega_{2} \\
& \delta A_{\alpha} \longmapsto \delta A_{2} .
\end{aligned}
$$

The scaling factor $(\lambda / r)^{2-\alpha}$ is expected to be $\leqslant 1$. For metrics without a horizon, the scale factor can be chosen. When there is a horizon, the scale factor is determined by its value on the horizon. To explore the numerical values of this factor in metrics with a horizon, a specific mass solution is needed.

\section{Applications}

\subsection{Constant mass solution}

A specific mass solution is necessary in order to explicitly treat diffusion and fractal behaviour. We have seen above that the sectional curvature mass can be written as

$$
2 M=\frac{1}{4} \alpha^{2}(2 m-r)+\lambda^{\alpha-2} r^{3-\alpha} .
$$

To illustrate the rescaling process, we explore the case of constant $M$. For $\alpha=2$ there is a Schwarzschild vacuum with a horizon at $r=2 m_{0}$. Assuming that all scales see the horizon at 
$2 m_{0}$ we can determine the scaling factor. From equation (24) with $M=m_{0}$ and $r=2 m=2 m_{0}$ it follows that

$$
\left(2 m_{0} / \lambda\right)^{\alpha-2}=1
$$

or $\lambda=2 m_{0}$.

With $m(r)$ from equation (24) the fluid parameters in equation (14) are

$$
\begin{aligned}
& 8 \pi \tilde{q}_{a}=0, \\
& 8 \pi \tilde{\rho}=\frac{6(\alpha-2)}{\alpha}\left(m_{0} r^{-3}\right), \\
& 8 \pi \tilde{p}_{r}=-\frac{2(\alpha-2)}{\alpha}\left[m_{0} r^{-3}+\lambda^{-2}(\lambda / r)^{\alpha}\right], \\
& 8 \pi p_{\perp}=\left(\frac{\alpha-2}{\alpha}\right)^{2}\left[-2 m_{0} r^{-3}+\lambda^{-2}(\lambda / r)^{\alpha}\right] .
\end{aligned}
$$

For this simple example, $\tilde{\rho}$, resulting from fluctuations, is negative [12] and there is no bounding $\tilde{p}_{r}=0$ surface. The radial profile describes an atmosphere asymptotically approaching a vacuum. If one examines the $\alpha<2$ metric, it appears not to be asymptotically flat. However, asymptotic flatness applies only to the observer's $\alpha=2$ spacetime.

\subsection{Solution with finite boundaries}

The $M=m_{0}$ solution is part of the homogeneous solution, equation (22). The complete homogeneous solution, modified to produce a vacuum Schwarzschild with a horizon at $r=2 m_{0}$ is

$$
M=m_{0}+m_{1}(2-\alpha)(r / \lambda)^{\alpha+1}
$$

with

$$
m=\frac{4 m_{0}}{\alpha^{2}}+\frac{4 m_{1}(2-\alpha)}{\alpha^{2}}(r / \lambda)^{\alpha+1}+\frac{r}{2}-\frac{2 \lambda}{\alpha^{2}}(r / \lambda)^{3-\alpha} .
$$

Evaluating this equation on the horizon at $r=2 m_{0}$ to find the scaling factors gives the equation, with $x=\lambda / 2 m_{0}$,

$$
1+\left(m_{1} / m_{0}\right)(2-\alpha) x^{-(\alpha+1)}-x^{\alpha-2}=0 .
$$

This more complex scaling equation has no solution for $m_{1} \geqslant m_{0}$, and may describe solutions without a horizon. For $m_{1} \ll m_{0}$ there are two scaling factors. Some typical values for $m_{1} / m_{0}=0.01$ are

$$
\begin{array}{rlrl}
\alpha & =\frac{7}{4}, & & x \simeq 1,1 / 7.6 \\
\alpha=\frac{6}{4}, & & x \simeq 1,1 / 11.9 \\
\alpha=\frac{5}{4}, & x \simeq 1,1 / 24.5 \\
\alpha=1, & x \simeq 1, \frac{1}{99} .
\end{array}
$$

For the complete homogeneous solution the fluid parameters are

$8 \pi \tilde{q}_{a}=0$,

$8 \pi \tilde{\rho}=\frac{2(\alpha-2)}{\alpha}\left[3 m_{0} r^{-3}+(4-5 \alpha)\left(m_{1} / \lambda^{3}\right)(r / \lambda)^{\alpha-2}\right]$, 


$$
\begin{aligned}
& 8 \pi \tilde{p}_{r}=-\frac{2(\alpha-2)}{\alpha}\left[m_{0} r^{-3}+\lambda^{-2}(\lambda / r)^{\alpha}-3 \alpha\left(m_{1} / \lambda^{3}\right)(r / \lambda)^{\alpha-2}\right], \\
& 8 \pi p_{\perp}=\left(\frac{\alpha-2}{\alpha}\right)^{2}\left[-2 m_{0} r^{-3}+\lambda^{-2}(\lambda / r)^{\alpha}\right]+\left(\frac{\alpha-2}{\alpha}\right)\left[(5 \alpha-4)\left(2 m_{1} / r^{3}\right)(r / \lambda)^{\alpha+1}\right] .
\end{aligned}
$$

For solution (27) there are boundaries for some $\alpha$. The boundary radii follow from $\tilde{p}_{r}=0$ at $\lambda=2 m_{0}$. With $y=r_{b} / 2 m_{0}$,

$$
1+2 y^{3-\alpha}-3 \alpha\left(m_{1} / m_{0}\right) y^{\alpha+1}=0 .
$$

Again choosing $m_{1} / m_{0}=0.01$, there are boundaries which become larger as $\alpha$ decreases:

$$
\begin{aligned}
& \alpha=\frac{7}{4}, \quad y \simeq 11.5 \\
& \alpha=\frac{6}{4}, \quad y \simeq 45 \\
& \alpha=\frac{5}{4}, \quad y \simeq 2800 \\
& \alpha=1, \quad \text { no boundary. }
\end{aligned}
$$

Since $\alpha=2$ is a vacuum solution, one could attempt to construct a matching vacuum for the lower values of $\alpha$. However, the lack of a boundary at $\alpha=1$ implies that the boundaries mark a change from pressure to tension rather than a boundary to vacuum. This supports a picture of an atmosphere which extends further as the size of the observed fluctuation decreases, all asymptotically approaching vacuum. We again remark that asymptotic flatness applies only to the observer's $\alpha=2$ spacetime.

\subsection{The Sierpinski gasket}

These ideas can be applied to diffusive processes whose continuum solutions are expected to envelope fractal behaviour at smaller scales. As an example, we will use some existing discussions of enveloped fractal structures in flat spaces to discuss the approximate sizes of $\alpha$ and $\beta$ where $D(r)=D_{0} r^{\beta-\alpha}=D_{0} r^{\Theta}$. The numerical parameters of interest are $\alpha$, the diffusivity factor $\Theta$, the Euclidean embedding dimension $d_{E}$ in which the fractal substrate is embedded and a fractal dimension $d_{F}$ (which may not be $d_{H}$ ). Alexander and Orbach [6] and O'Shaughnessy and Procaccia [5] provide some numerical values of $d_{F}$ and $\Theta$ for various fractal processes. The fractal dimension $d_{F}$ is defined through the diffusion equation which is written as

$$
\frac{\partial \Gamma}{\partial u}=\frac{1}{r^{d_{F}-1}} \frac{\partial\left(D_{0} r^{-\Theta} r^{d_{F}-1} \partial_{r} \Gamma\right)}{\partial r} .
$$

In $d_{E}=3$, the fractal dimension for the Sierpinski gasket is $d_{F}=2$ and $\Theta=0.59$. The corresponding values of $\alpha$ and $\beta$ are $\alpha=1, \beta=1.585$. For $d_{E}=4$, the fractal dimension is $d_{F}=2.31$ and $\Theta=0.81$. We find $\alpha=1.32$ and $\beta=2.13$.

For all of the above examples, we could find a $u$-independent solution of the diffusion equations as

$$
\begin{aligned}
& \Gamma=\Gamma_{0} r^{1-\beta}, \\
& M=M_{0}+M_{1} r^{\alpha+1} .
\end{aligned}
$$

The mass scaling is set entirely by the metric parameter $\alpha$, while the density depends on the scaling of the diffusivity. 


\section{Conclusion}

Introducing a parameter, $\alpha$, into the extended Schwarzschild metric, produces an atmosphere with processes that can be linked to a fractal mass distribution. Specific values of $\alpha$ can be associated with particular fractal substrates in a small-scale region of the atmosphere. The continuous solutions of an anomalous diffusion equation in the atmosphere envelope smallerscale fractal processes. Anomalous diffusion can sample fractal processes on a topological level, giving information about the connectivity as well as the fractal dimension. Fluid parameters from the field equations can be studied for a range of $\alpha$, allowing the effects of fluctuations from one scale to another to be examined.

Other models are possible. For example, a fractal Schwarzschild cell could be used in a universe model such as the Lindquist-Wheeler model [13], perhaps describing the fractal galactic density distribution. If the galactic distribution can be modelled by a single fractal process in which both mass and density diffuse, then on time scales that are short compared with the diffusion time, the mass would scale with the fractal dimension, while the scaling of the density would involve topological elements of the fractal substrate. In this paper only a single level of scale reduction has been studied. It is possible that an ensemble of solutions, a multifractal set summed over $\alpha$, could be used to model more complex physical systems.

Another direction for future work is a possible link between fractal atmospheric structure and chaos. A variety of perturbations to a black hole solution have been shown to lead to chaotic behaviour, most often chaotic particle orbits [14]. For example, Bombelli and Calzetta [15] have shown that gravitational perturbations of the Schwarzschild solution can produce chaotic orbits. In general, the effects of chaos range from unusual production of gravitational waves [16] to a possible brightening of the black hole [17]. Orbiting particles with spin may also exhibit chaotic behaviour [18, 19]. A relation between the phase space fractal dimension and chaos has been established for multi-black-hole spacetimes [20] and for black-hole chaotic scattering processes $[21,22]$. We have considered an atmosphere that has fractal structure in the physical spacetime. At one scale, the solution can describe an ideal black hole, or with time dependence and space dependence, a black hole with a two-fluid atmosphere. The effect of perturbations of this atmosphere and the effects of the physical fractal structure on particle orbits is an interesting area for investigation.

\section{References}

[1] Vaidya P C 1953 Nature 171260

[2] Glass E N and Krisch J P 1999 Class. Quantum Grav. 161175

[3] Ghez R and Langlois W E 1986 Am. J. Phys 54646

[4] Glass E N and Krisch J P 2000 Gen. Rel. Grav. 32735

[5] O'Shaughnessy B and Procaccia I 1985 Phys. Rev. Lett. 54455

[6] Alexander S and Orbach R 1982 J. Phys. Lett. 43625

[7] Dekeyser R, Maritan A and Stella A L 1994 Diffusion processes: experiment, theory, simulations Proc. 5th Max Born Symp. (Berlin: Springer)

[8] Nelson E 1985 Quantum Fluctuations (Princeton, NJ: Princeton University Press)

[9] Misner C W and Sharp D H 1964 Phys. Rev. B 136571

[10] Hughes B D 1995 Random Walks and Random Environments vol 1 (Oxford: Oxford University Press)

[11] Hastings H M and Sugihara G 1994 Fractals—a User's Guide for the Natural Sciences (Oxford: Oxford University Press)

[12] Ford L H and Roman T A 1997 Phys. Rev. D 552082

[13] Lindquist R W and Wheeler J A 1957 Rev. Mod. Phys. 29432

[14] Rugh S E 1994 Chaos in the Einstein equations-characterization and importance Deterministic Chaos in General Relativity ed D Hobill, A Burd and A Coley (New York: Plenum)

[15] Bombelli L and Calzetta E 1992 Class. Quantum Grav. 92573 
[16] Cornish N J and Franke N E 1997 Phys. Rev. D 561903

[17] Levin J 1999 Phys. Rev. D 60064015

[18] Suzuki S and Maeda K 1997 Phys. Rev. D 554848

[19] Suzuki S and Maeda K 2000 Phys. Rev. D 61024005

[20] Dettmann C P, Frankel N F and Cornish N J 1994 Phys. Rev. D 50 R618

[21] Frolov A V and Larsen A L 1999 Class. Quantum Grav 163717

[22] deMoura A P S and Letelier P S 1999 Chaos and fractals in geodesic motions around a non-rotating black hole with an external halo 1999 Preprint chao-dyn/9910035 